\title{
Unprinted Materials
}

\section{Archives of the International Labour Office}

Given the considerable number of ILO files consulted, only the titles of the funds are given. The reader may refer to the detailed quotations in the book.

Cabinet Files:

Albert Thomas papers

Harold Butler papers

Cabinet John G. Winant + Edward

Phelan papers

David Morse papers

Private papers of Wilfred Jenks

ILO Division and Technical Services files:

Series C: Correspondent

Series CO: Cooperatives

Series D: Diplomatic Division

Series E: Emigration

Series EP: "Economic problems"

Series F: Finance

Series FO: Field Office

Series FS: Fellowships

Series G: General

Series GB: Governing Body

Series HY: Hygiene

Series LBO: London Branch Office

Series LE: Legislation

Series MI: Missions

Series MP: Manpower

Series N: Trade Union Movement

Series RL: Relations

Series RRA: United Nations Relief and

Rehabilitation administration

Series SI: Social insurance

Series T: Statistics

Series TA: Technical assistance

\author{
Personal files (Series P): \\ Harold Butler, P 7 \\ Camille Pône, P 9 \\ Maurice Thudichum, P 14 \\ Percival Martin, P 19 \\ Roger Plissard, P 82 \\ Harold Grimshaw, P 131 \\ Royal Meeker, P 192 \\ Adrien Tixier, P 217 \\ Luigi Carozzi, P 506 \\ Christie Tait, P 752 \\ David Blelloch, P 760 \\ Maurice Stack P1188 \\ Fernand Maurette, P 1720 \\ Oswald Stein, P 1289 \\ Carlos Garcia-Palacios, P1299 \\ Cyrille Dechamp, P 1729 \\ Xavier Bueno, P 1848 \\ Charles Becker, P 1947 a) \\ John Edward Riches, P 1998 \\ Moisés Poblete Troncoso, P 2037 \\ Enrique Siewers, P 2122 \\ Jaroslav Drbohlav, P 2231 \\ Wilfred Jenks, P 2597 \\ Hans Staehle, P 2446 \\ Ryoichi Kojima, P 2942 \\ Anton Zelenka, P 3611 \\ Emil Schoenbaum, P 3926
}

\section{Archives of the League of Nations, Palais des Nations, Geneva}

ONUG, ASDN, R 3995, 5B/5867/1226: Collaboration of the Government of China the work of Intellectual Cooperation. Discussion at the 15th session of the CICl. July 1933. 
United Nations Archives, Palais des Nations, Geneva

ONUG, G.X. “ Affaires économiques” (CEE), 18/11/1/1 “Manpower. EECE Documentation. Study by ECE, IRO, and ILO concerning Manpower position in Europe".

\section{Archives of the International Institute for Intellectual Cooperation (UNESCO), Paris}

AUNESCO, AG 1 - International Institute of Intellectual Co-operation, IICI, Al (Direction), 135 (Relations with the Government of China), 1932-1946.

AUNESCO, AG 1 - International Institute of Intellectual Co-operation, IICl, Alll (Correspondence), 55 (China), 1933-1946.

\section{United Nations Archives and Records Centre (ARC), New York, USA}

ARC, United Nations Relief and Rehabilitation Administration UNRRA, Lake Success Office, S1303-0000-5485 (1943-1949); S-1303-0000-5486 (1943-1949); S-1303-00005487 (1943-1949); S-1303-0000-5488 (1943-1949); S-1303-0000-5489 (1943-1949).

\section{Rockefeller Archive Center, Pocantico Hills, New York, USA}

RG 1.1 (Projects), Series $100 \mathrm{~S}$ (International - Social Sciences):

- Box 108, Folder 973: International Labor Office, 1936-1940.

- Box 108, Folder 974: International Labor Office - Armament Program Study, 1939-1941.

- Box 108, Folder 975: International Labor Office - Armament Program Study, 1939-1941.

- Box 108, Folder 977: International Labor Office - Migration, 1941-1942.

- Box 108, Folder 978: International Labor Office - Migration, 1941-1942.

- RG 1.2 (Projects), Series 100 E (International - Fellowships, Scholarships):

- Box 35, Folder 260: Training Awards, (Fellowships - Administration and Policy) - Stipend, $1935-1961$.

Collection NAR, Record Groupe (RG) 4, NELSON A. ROCKEFELLER, PERSONAL PAPERS. Series: O, Washington DC, Sub-Series IAA:

- Box 1, Folder 1 and 2: Washington DC files, CIAA. General, 1940.

- Box 1, Folder 3: Washington DC files, CIAA. General, 1942.

- Box 1, Folder 4: Washington DC files, CIAA. General, 1943.

- Box 1, Folder 5: Washington DC files, CIAA. General, 1944.

- Box 3, Folders 18-24: Latin America post-war planning, 1941-1945.

Collection NAR, Record Groupe (RG) 4, NELSON A. ROCKEFELLER, PERSONAL PAPERS. Series: A, Personal Activities:

- Box 144, folders 1565-1573: Trips South America. Itineraries, Guest lists, 1937). 
National Archives and Records Administration (NARA), College Park, Maryland, USA

RG 174: General records of the Department of Labor, 1907-1986

174.3.1 Records of Secretaries.

- Box 45: Office of the Secretary. Secretary Frances Perkins. General Subject File, 1933-1941. Conferences.

- Box 46: Office of the Secretary. Secretary Frances Perkins. General Subject File, 1933-1941. Conferences.

- Box 50: Office of the Secretary. Secretary Frances Perkins. General Subject File, 1933-1941. Conferences to Embargo.

- Box 74: Office of the Secretary. Secretary Frances Perkins. General Subject File, 1933-1941. International Labor Office.

- Box 75: Office of the Secretary. Secretary Frances Perkins. General Subject File, 1933-1941. International Labor Office to Japanese Trade Union Congress.

B- ox 92: Office of the Secretary. Secretary Frances Perkins. General Subject File, 1933-1941. Resettlement Administration to Social Security Board.

Columbia University Rare Books and Manuscript Library, New York City, USA

Lewis Lorwin Papers.

Seeley G. Mudd Rare Books and Manuscript Library, Princeton, USA

David Morse Papers.

Harvard Law School Library \& Historical and Special Collections, Cambridge, USA

Calvert Magruder Papers.

Leeds University Library Special Collections, Leeds, UK

David Blelloch Papers.

Columbia Center for Oral History, Butler Library, Columbia University, New York, USA

Oral History interviews with David A. Morse, Oral History Research Office, Columbia University 1981. Conducted par Peter Jessup, Columbia University, Washington DC, 19 July, 25 July, 2 August, 9 August, 11 October, 25 October 1980 and 11 January, 7 March 1981. 


\section{Private Records}

Personal Papers of Maurice Thudichum, held by is grand-son Pierre Roehrich, Geneva. 